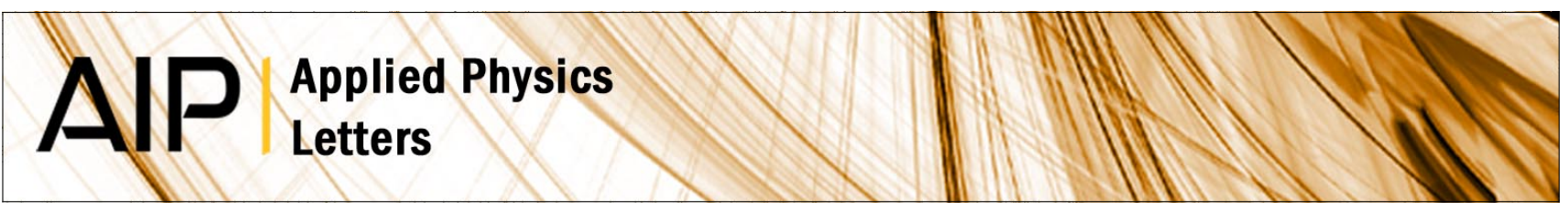

\title{
An insight into voltage-biased superconducting quantum interference devices
}

Chao Liu, Yi Zhang, Michael Mück, Hans-Joachim Krause, Alex I. Braginski et al.

Citation: Appl. Phys. Lett. 101, 222602 (2012); doi: 10.1063/1.4768698

View online: http://dx.doi.org/10.1063/1.4768698

View Table of Contents: http://apl.aip.org/resource/1/APPLAB/v101/i22

Published by the American Institute of Physics.

\section{Additional information on Appl. Phys. Lett.}

Journal Homepage: http://apl.aip.org/

Journal Information: http://apl.aip.org/about/about_the_journal

Top downloads: http://apl.aip.org/features/most_downloaded

Information for Authors: http://apl.aip.org/authors

\section{ADVERTISEMENT}

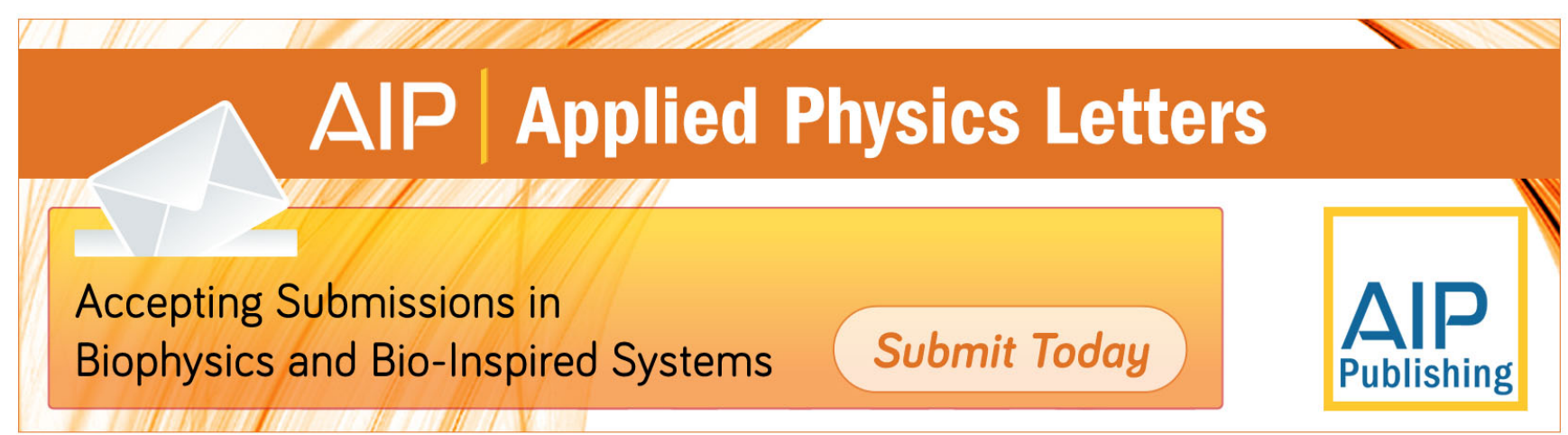




\title{
An insight into voltage-biased superconducting quantum interference devices
}

\author{
Chao Liu, ${ }^{1,2,3,4}$ Yi Zhang, ${ }^{2,3, a)}$ Michael Mück, ${ }^{5}$ Hans-Joachim Krause, ${ }^{2,3}$ Alex I. Braginski, ${ }^{2}$ \\ Xiaoming Xie, ${ }^{1,3}$ Andreas Offenhäusser, ${ }^{2,3}$ and Mianheng Jiang ${ }^{1,3}$ \\ ${ }^{1}$ State Key Laboratory of Functional Materials for Informatics, Shanghai Institute of Microsystem and \\ Information Technology (SIMIT), Chinese Academy of Sciences (CAS), Shanghai 200050, \\ People's Republic of China \\ ${ }^{2}$ Peter Grünberg Institute (PGI-8), Forschungszentrum Jülich (FZJ), D-52425 Jülich, Germany \\ ${ }^{3}$ Joint Research Laboratory on Superconductivity and Bioelectronics, Collaboration between CAS-Shanghai, \\ Shanghai 200050, People's Republic of China and FZJ, D-52425 Jülich, Germany \\ ${ }^{4}$ Graduate University of the Chinese Academy of Sciences, Beijing 100049, China \\ ${ }^{5}$ Institute of Applied Physics, University of Gießen, D-35392 Giessen, Germany
}

(Received 2 October 2012; accepted 8 November 2012; published online 30 November 2012)

\begin{abstract}
We experimentally studied two important parameters of helium-cooled superconducting quantum interference devices (SQUIDs) in the voltage bias mode: the dynamic resistance $R_{\mathrm{d}}$ and the flux-tocurrent transfer coefficient $\partial i / \partial \Phi$, with different junction shunt resistors $R_{\mathrm{J}}$. We investigated a voltage-biased SQUID using the direct readout current-to-voltage converter scheme involving an operational amplifier. At higher $R_{\mathrm{J}}$, the flux-to-voltage conversion coefficient $\partial V / \partial \Phi$ becomes sufficiently large to effectively suppress the room-temperature amplifier's noise without any need for additional feedback circuits. The McCumber parameter limits the rise of $\partial V / \partial \Phi$. We discuss the performance of voltage-biased SQUIDs at different effective McCumber parameters. (C) 2012 American Institute of Physics. [http://dx.doi.org/10.1063/1.4768698]
\end{abstract}

In most cases, direct current superconducting quantum interference devices (dc SQUIDs) are operated in the current-biased mode. In this mode, the SQUID behavior can be well interpreted by theory, and parameters such as the McCumber parameter $\beta_{\mathrm{c}}{ }^{1,2}$ and the screening parameter $\beta_{\mathrm{L}}{ }^{3}$ can be determined from simulations. In voltage bias mode, it is much more difficult to simulate SQUID operation and to determine the parameters for optimum performance. The voltage-biased SQUID is connected to a voltage source, and the current flowing in the circuit is modulated by the external magnetic flux, with a period of $\Phi_{0}$, the flux quantum. We note that the SQUID intrinsic noise is independent of the selected bias mode. ${ }^{4}$

In contrast to a current source, which is fairly easy to realize as a SQUID exhibits a low impedance of a few tens of ohms at most, making a voltage source with an internal resistance $R_{\text {in }} \approx 0$ is very difficult. To amend this problem, two SQUID readout schemes approximating an ideal voltage source have been proposed, the two-stage SQUID $^{5}$ and the current-to-voltage converter (CVC) using an operational amplifier. ${ }^{6}$ In the two-stage-SQUID scheme, the SQUID is read out by a second SQUID, thus avoiding additional noise from the room-temperature semiconductor amplifier. However, due to the necessity to bias two SQUIDs, this scheme is difficult to use and is thus not often employed in practical applications. ${ }^{4}$

In this paper, we statistically study two important SQUID parameters, the nonlinear dynamic resistance $R_{\mathrm{d}}$ and the current-to-flux transfer coefficient $\partial i / \partial \Phi$ of a SQUID operated in the voltage-biased mode using the CVC scheme. To obtain meaningful results, we performed the measurements

\footnotetext{
${ }^{\text {a) }}$ Author to whom correspondence should be addressed. Electronic mail: y.zhang@fz-juelich.de.
}

on a large number of SQUIDs $(\sim 100)$. We find that the voltage-biased SQUID offers an important technical advantage over the current-bias scheme. With an appropriately high junction shunt resistance, $R_{\mathrm{J}}$, this SQUID can reach a low noise level even when it is directly connected to a roomtemperature operational amplifier. Furthermore, we report the voltage-biased-SQUID performance at different $R_{\mathrm{J}}$.

For our experiments, we prepared helium-cooled planar SQUID magnetometers with SQUID-loop inductance of $L_{\mathrm{s}}=350 \mathrm{pH}$. The magnetometers consist of a parallel gradiometer SQUID with two integrated oppositely wound input coils, $L_{\text {in }}$, connected in series with the on-chip pickup loop $L_{\mathrm{p}}$. The SQUIDs with a chip size of $5 \times 5 \mathrm{~mm}^{2}$ were fabricated in conventional niobium technology. Four different planar junction shunt resistors $R_{\mathrm{J}}$ of nominally $5,10,30$, and $40 \Omega$ were designed. The flux-to-field coefficient $\partial B / \partial \Phi$ of the magnetometers was measured to be $1.5 \mathrm{nT} / \Phi_{0}$. Two planar coils, $L_{1}$ and $L_{2}$, which can be used to implement suitable current and voltage feedbacks of SQUID bootstrap circuitry (SBC), ${ }^{7}$ were also integrated in the design as an option. The coil $L_{\text {FLL }}$ was integrated on the chip for the operation in fluxlocked loop mode.

The current-biased dc SQUID will convert a change in the input flux to a change in the voltage across the SQUID, while the voltage-biased SQUID will convert a flux change to a current change in the SQUID and its external circuitry. In the latter case, the two essential parameters, $R_{\mathrm{d}}=\partial V / \partial i$ and the flux-to-current transfer coefficient $\partial i / \partial \Phi$, are determined by the $I-V$ and $I-\Phi$ characteristics of the SQUID at the working point. The voltage-biased SQUID is directly connected to the inverting input of an operational amplifier, while the bias voltage is applied at its non-inverting input (see the inset of Fig. 1). The operational amplifier has two noise sources, the 


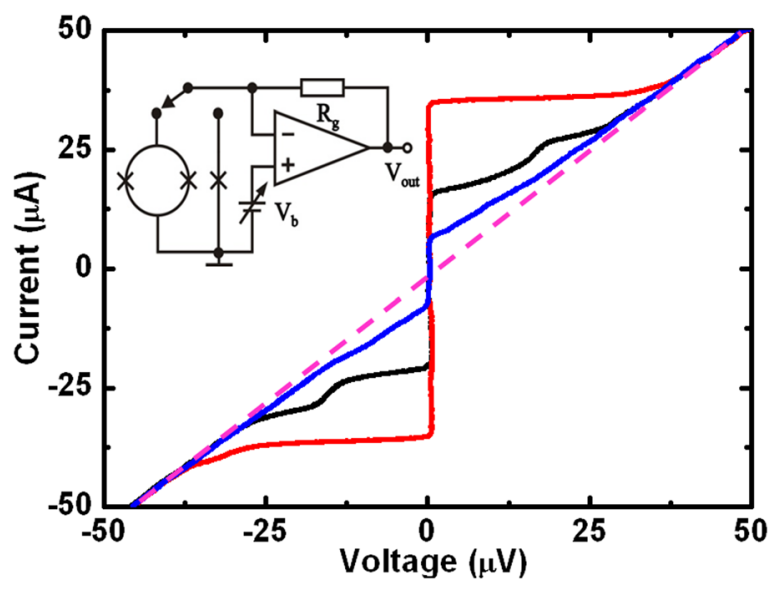

FIG. 1. Current-voltage characteristics of three junctions with different $I_{\mathrm{c}}$. Here, the source resistances $R_{\text {in }}$ have been taken out with data processing. Inset depicts the current-to-voltage converter scheme used in this work.

voltage noise $V_{\mathrm{n}}$, and the current noise $I_{\mathrm{n}}{ }^{*}$, which must be taken into account. The total current noise $I_{\mathrm{n}}$ flowing from the preamplifier output is $I_{\mathrm{n}}=\left[\left(V_{\mathrm{n}} / R_{\mathrm{d}}\right)^{2}+I_{\mathrm{n}}{ }^{* 2}\right]^{1 / 2}$, where $R_{\mathrm{d}}$ denotes the dynamic resistance of the SQUID. The equivalent flux noise of the preamplifier is $\delta \Phi_{\mathrm{amp}}=I_{\mathrm{n}} /(\partial i / \partial \Phi)$. Generally, the contribution of $I_{\mathrm{n}}{ }^{*}$ is independent of $R_{\mathrm{d}}$ and can be ignored in the voltage bias mode. ${ }^{8}$ Consequently, the expression can be simplified to $\delta \Phi_{\mathrm{amp}}=\left(V_{\mathrm{n}} / R_{\mathrm{d}}\right) /(\partial i / \partial \Phi)$ $=V_{\mathrm{n}} /(\partial V / \partial \Phi)$. The voltage-bias parameter $\partial V / \partial \Phi$ is a product of $R_{\mathrm{d}}$ and $\partial i / \partial \Phi$. In our experiments, we used the low noise operational amplifier Analog Devices 797 with a typical voltage noise of $V_{\mathrm{n}} \approx 1 \mathrm{nV} / \sqrt{ } \mathrm{Hz}$ in the white noise range $(>100 \mathrm{~Hz})$.

It is illustrative to first measure junctions in the CVC scheme. Figure 1 shows measured $I-V$ characteristics of single junctions with different critical currents $I_{\mathrm{c}}$. Each junction was shunted by the resistance $R_{\mathrm{J}}=1 \Omega$. The dashed line describes the shunted junction's normal resistance $R_{\mathrm{N}} \approx R_{\mathrm{J}}$. In the non-linear regime, i.e., at low voltages $R_{\mathrm{d}}$ increases with increasing $I_{\mathrm{c}}$. The $I_{\mathrm{c}}$ values were measured to be about $6.4,15.5$, and $34 \mu \mathrm{A}$, which resulted in dynamic resistances $R_{\mathrm{d}} \approx 1.2 \Omega, 3 \Omega$, and $25 \Omega$, respectively.

According to theory, ${ }^{9}$ a critical current $I_{\mathrm{c}}$ of about $\Phi_{0} / 2 L_{\mathrm{s}}$ is needed to obtain the optimum SQUID screening parameter of $\beta_{\mathrm{L}} \approx 1$. We investigated the dependence of $R_{\mathrm{d}}$ and $\partial i / \partial \Phi$ upon $R_{\mathrm{J}}$ using different junction shunt resistors $R_{\mathrm{J}}$ of 5,10 , 30 , and $40 \Omega$. Overall, 101 SQUID magnetometers were measured to obtain the averaged data illustrated in Fig. 2. The statistical data showed that $R_{\mathrm{J}}$ increasing from $5 \Omega$ to $40 \Omega$ resulted in SQUID current swing $I_{\text {swing }}$ decreasing from $4.6 \mu \mathrm{A}$ to $2.9 \mu \mathrm{A}$ (a), which corresponded to $\partial i / \partial \Phi$ from $14 \mu \mathrm{A} / \Phi_{0}$ to $9 \mu \mathrm{A} / \Phi_{0}$, while $R_{\mathrm{d}}$ increased from $6.5 \Omega$ to $44 \Omega$ (b). The product $(\partial i / \partial \Phi) \times R_{\mathrm{d}}=\partial V / \partial \Phi$ increased up to about $400 \mu \mathrm{V} / \Phi_{0}$ at $R_{\mathrm{J}}=30$ to $40 \Omega$. High $R_{\mathrm{J}}$ leads to a large $\partial V /$ $\partial \Phi$, thus reducing the preamplifier noise contribution $\delta \Phi_{\mathrm{amp}}$. A large $R_{\mathrm{J}}$ is also required for a low SQUID intrinsic flux noise spectral density, which is $S_{\Phi}=16 k_{\mathrm{B}} T L_{\mathrm{S}}^{2} / \mathrm{R}_{\mathrm{J}}$ at the optimal $\beta_{\mathrm{L}} \approx 1$, where $k_{\mathrm{B}}$ denotes the Boltzmann constant and $T=4.2 \mathrm{~K}$.

Deviations of the SQUID parameters from the design values due to variations in the fabrication processes cannot be avoided. In our case, the maximum-to-minimum ratios of the two parameters shown in Fig. 2 were rather large, e.g., in the case of $R_{\mathrm{J}}=30 \Omega$, the ratios were about 2 for $I_{\text {swing }}$ and 3 for $R_{\mathrm{d}}$. The SQUIDs intrinsic noise does not depend on these two parameters directly, but $\delta \Phi_{\mathrm{amp}}$ in direct readout electronics does. As shown below, adding an additional feedback circuitry helps to overcome the drawbacks of large parameter spreads.

Measurements of noise at different $R_{\mathrm{J}}$ values were performed in a flux-locked loop, while the SQUID magnetometer chip, whose equivalent circuit is shown in the inset of Fig. 3, was placed in a niobium tube. We used our direct readout $\mathrm{CVC}$ scheme with the working point selected at maximum $\partial i / \partial \Phi$ in measured $I-\Phi$ characteristics.

Figure 3 shows noise measurements of SQUIDs with different $R_{\mathrm{J}}$ : (I) $5 \Omega$, (II) $10 \Omega$, and (III) $30 \Omega$. The measured flux noise decreased from $17 \mu \Phi_{0} /{ }_{\sqrt{ }} \mathrm{Hz}$ (I) to $13 \mu \Phi_{0} /{ }_{\sqrt{ }} \mathrm{Hz}$ (II) and $4 \mu \Phi_{0} / \sqrt{ } \mathrm{Hz}$ (III). The lowest value corresponds to a field resolution $\sqrt{\sqrt{ }} \mathrm{S}_{\mathrm{B}} \approx 6 \mathrm{fT} /{ }_{\sqrt{ }} \mathrm{Hz}$ in the white noise range. Clearly, $\partial V / \partial \Phi$ increases with $R_{\mathrm{J}}$ so that $\delta \Phi_{\text {amp }}$ is increasingly suppressed. We note that here the measured noise was mainly determined by the voltage noise of the room-temperature preamplifier rather than by the SQUID itself.

We then used the additional feedback circuitry, SBC, ${ }^{7}$ which is already integrated on our SQUID chip, to further reduce $\sqrt{ } S_{\Phi}$. Using the SBC readout scheme, the flux noise of SQUID (II) reached about $3 \mu \Phi_{0} / \sqrt{ } \mathrm{Hz}\left({ }_{\sqrt{ }} S_{\mathrm{B}}<5 \mathrm{fT} /{ }_{\sqrt{ }} \mathrm{Hz}\right)$, as shown in Fig. 3 IV. In the case of larger $R_{\mathrm{J}} \geq 30 \Omega$, the noise of some SQUIDs with smaller $\partial V / \partial \Phi$ resulting from the parameter spread in fabrication was also improved to this level (data not shown here).

Generally, a junction Stewart-McCumber parameter $\beta_{\mathrm{c}} \equiv 2 \pi I_{\mathrm{c}} R_{\mathrm{J}}{ }^{2} C / \Phi_{0}<1$ is required for SQUID operation in
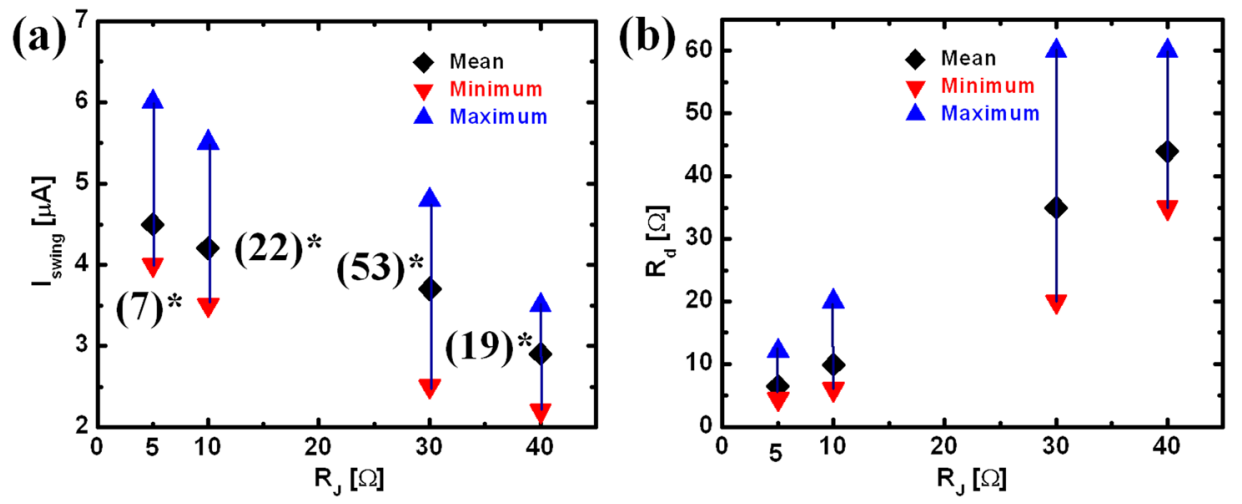

FIG. 2. Statistical data of $I_{\text {swing }}$ and $R_{\mathrm{d}}$ of 101 voltage-biased SQUIDs as a function of $R_{\mathrm{J}}$ (..)* denotes the number of samples. 


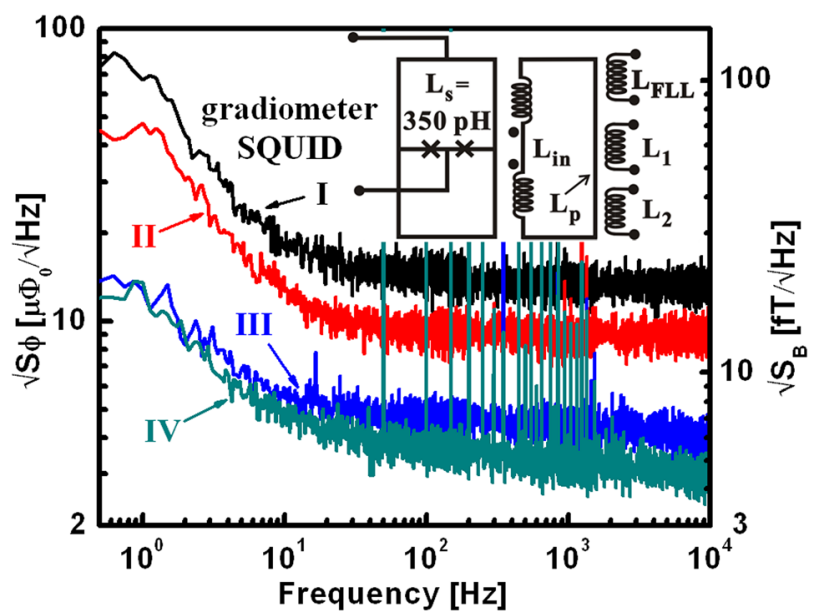

FIG. 3. Flux noise ${ }_{\mid} S_{\Phi}$ measurements (right ordinate gives the field resolution $\left.S_{\mathrm{B}}\right)$ of SQUIDs with different $R_{\mathrm{J}}$ operated in voltage bias mode using the $\mathrm{CVC}$ readout scheme. The inset shows the equivalent circuit of the measured magnetometers described above.

both current and voltage bias mode; here, $C$ denotes the junction's self-capacitance. The source resistance $R_{\text {in }}$ is parallel to $R_{\mathrm{J}}$ and shunts it in the voltage bias mode. From simulations by Warman and Blackburn, ${ }^{10}$ it is known that with decreasing ratio of source and junction resistance, $R_{\mathrm{in}} / R_{\mathrm{J}}$, the junction hysteresis disappears and eventually a negative dynamic resistance appears. For SQUIDs biased by a source of sufficiently small $R_{\text {in }}$, its shunting effect, which leads to effective $\beta_{\mathrm{ce}}<\beta_{\mathrm{c}}$, was first reported by Zhang et al. ${ }^{11}$ At $\beta_{\mathrm{ce}}>1$, a negative resistance appears in SQUID characteristics, which can lead to instabilities or oscillations in the readout circuit. The enhancement of SQUIDs $\partial V / \partial \Phi$ with increasing $R_{\mathrm{J}}$ is limited by the requirement that $\beta_{\text {ce }}$ remains less than one.

It is known that the SQUID $I-V$ and $V-\Phi$ characteristics both exhibit hysteresis at $\beta_{\mathrm{c}}>1$ in current bias mode. The value of $\beta_{\mathrm{c}}$ can be estimated from the amount of hysteresis observed in the characteristics. Here, we report on the voltage-biased SQUID performance in the range from $\beta_{\text {ce }}<1$ to $\beta_{\text {ce }} \gg 1$ using the $I-\Phi$ characteristic as our test signal. Figure 4 shows measured $I-\Phi$ characteristics of SQUIDs with different $R_{\mathrm{J}}$ values. In Fig. $4(\mathrm{a}), R_{\mathrm{J}}=10 \Omega$, the $I$ - $\Phi$ characteristics exhibits a quasi-triangular-shaped curve without any distortion and a large current swing. Its average value is $I_{\text {swing }}=4.2 \mu \mathrm{A}$. In this case, $\delta \Phi_{\text {amp }}$ can be effectively reduced by using SBC, as shown in Fig. 3 IV. We infer this case corresponds to $\beta_{\mathrm{ce}}<1$. At higher nominal values of $R_{\mathrm{J}}$ $\left(R_{\text {in }}=\right.$ constant $)$, we observe increasing distortions and oscillations in the $I-\Phi$ characteristics. In Fig. 4(b), nominal $R_{\mathrm{J}}=40 \Omega$, at the bottom of the $I-\Phi$ curve a distortion and oscillation are visible. The inferred $\beta_{\text {ce }}$ is $\approx 1$. In this SQUID, the intrinsic noise is higher than that in SQUID (a); the measured data are not shown here. In Fig. 4(c), with $R_{\mathrm{J}}$ nominally also $40 \Omega$, the oscillation appears at most parts of the $I-\Phi$ curve, and measured noise is still higher, so presumably $\beta_{\text {ce }}>1$. In Fig. 4(d), for the case of $R_{\mathrm{J}}=200 \Omega$, a strong oscillation dominates the whole $I-\Phi$ dependence; here, we clearly have $\beta_{\text {ce }} \gg 1$.

All our voltage-biased SQUIDs with $R_{\mathrm{J}}=30 \Omega$ operated very stably and exhibited a good noise performance, even without SBC. The $I-\Phi$ traces were as in Fig. 4(a).

In conclusion, our experimental and statistical investigation has shown that the SQUID direct readout current-tovoltage converter scheme permits one to largely suppress the noise contribution of the room-temperature operational amplifier. To achieve this, the junction shunt resistance should be appropriately high, but the effective McCumber parameter less than 1. Consequently, provided that fabrication of SQUIDs with tight parameter spreads is attained, it should be possible to use the direct readout CVC scheme without any additional feedbacks and further simplify the readout. In the case of wide parameter spreads, a feedback scheme, such as the SQUID bootstrap circuit, is useful in effectively suppressing the noise contribution of the amplifier.

These results help to understand SQUIDs operated in voltage bias mode and can be used to develop very simple multi-channel SQUID systems, e.g., for biomagnetic measurements.
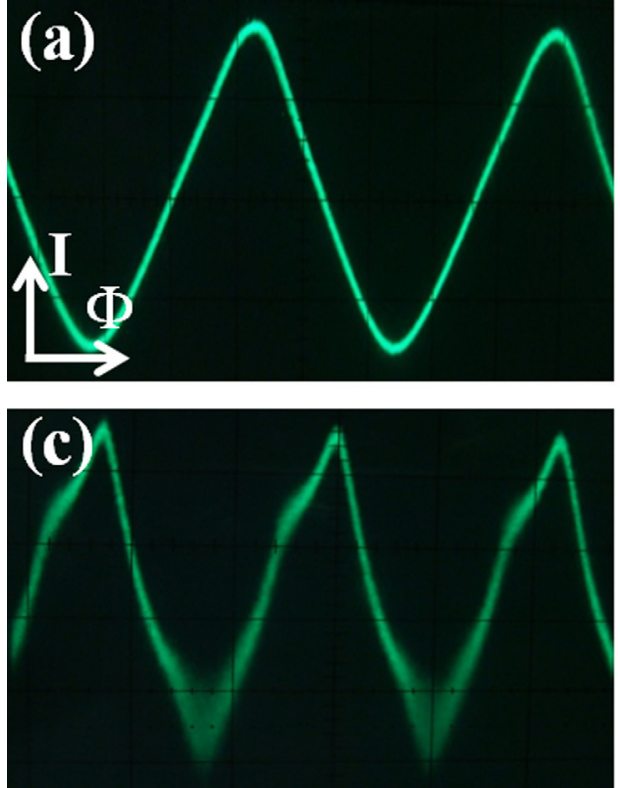
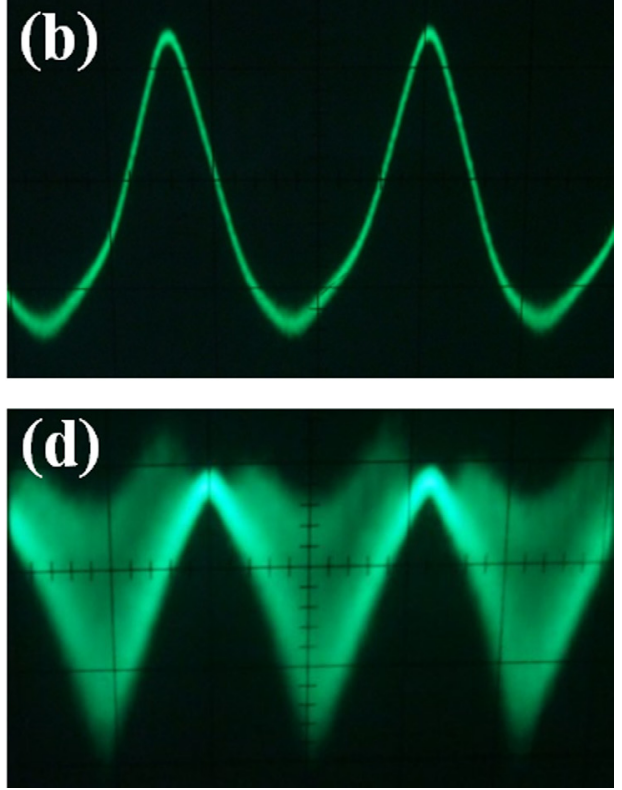

FIG. 4. I- $\Phi$ characteristics at $\beta_{\mathrm{ce}}<1$ (a), $\beta_{\mathrm{ce}} \approx 1$ (b), $\beta_{\mathrm{ce}}>1$ (c), and $\beta_{\mathrm{ce}} \gg 1$ (d). 
The authors thank Dr. Zhen Wang, Dr. Guofeng Zhang, and Mr. Yongliang Wang for the help to measure the $I-V$ characteristics of junctions.

${ }^{1}$ W. C. Stewart, Appl. Phys. Lett. 12, 277-280 (1968).

${ }^{2}$ D. E. McCumber, J. Appl. Phys. 39, 3113-3118 (1968).

${ }^{3}$ J. Clarke, in The SQUID Handbook, edited by J. Clarke and A. I. Braginski (Wiley-VCH, Weinheim, 2004), Vol. I, pp.1-28.

${ }^{4}$ D. Drung and M. Mück, in The SQUID Handbook, edited by J. Clarke and A. I. Braginski (Wiley-VCH, Weinheim, 2004), Vol. I, pp.127-170.

${ }^{5}$ F. C. Wellstood, C. Urbina, and J. Clarke, Appl. Phys. Lett. 50, 772 (1987).
${ }^{6}$ H. Seppä, A. Ahonen, J. Knuutila, J. Simola, and V. Vilkman, IEEE Trans. Magn. 27, 2488-2490 (1991).

${ }^{7}$ X. Xie, Y. Zhang, H. Wang, Y. Wang, M. Mück, H. Dong, H.-J. Krause, A. I. Braginski, A. Offenhäusser, and M. Jiang, Supercond. Sci. Technol. 23, 065016 (2010).

${ }^{8}$ G. Zhang, Y. Zhang, H. Dong, H.-J. Krause, X. Xie, A. I. Braginski, A. Offenhäusser, and M. Jiang, Supercond. Sci. Technol. 24, 065023 (2011). ${ }^{9}$ C. D. Tesche and J. Clarke, J. Low Temp. Phys. 29, 301-331 (1977).

${ }^{10}$ J. Warman and J. A. Blackburn, Appl. Phys. Lett. 19, 60 (1971).

${ }^{11}$ Y. Zhang, C. Liu, M. Schmelz, H.-J. Krause, A. I. Braginski, R. Stolz, X. Xie, H.-G. Meyer, A. Offenhäusser, and M. Jiang, Supercond. Sci. Technol. 25, 125007 (2012). 